\title{
Efficacious proteasome/HDAC inhibitor combination therapy for primary effusion lymphoma
}

\author{
Shruti Bhatt, ${ }^{1,2}$ Brittany M. Ashlock, ${ }^{3,4}$ Ngoc L. Toomey,, Luis A. Diaz,2 \\ Enrique A. Mesri,3,4 Izidore S. Lossos,1,2 and Juan Carlos Ramos'2,3,4

\begin{abstract}
1Department of Molecular and Cellular Pharmacology, ${ }^{2}$ Department of Medicine, Division of Hematology-Oncology, ${ }^{3}$ Viral Oncology Program, Sylvester Comprehensive Cancer Center, and ${ }^{4}$ Center for AIDS Research and Department of Microbiology and Immunology, University of Miami Miller School of Medicine, Miami, Florida, USA.
\end{abstract}

\begin{abstract}
Primary effusion lymphoma (PEL) is a rare form of aggressive B cell lymphoma caused by Kaposi's sarcomaassociated herpesvirus (KSHV). Current chemotherapy approaches result in dismal outcomes, and there is an urgent need for new PEL therapies. Previously, we established, in a direct xenograft model of PELbearing immune-compromised mice, that treatment with the proteasome inhibitor, bortezomib (Btz), increased survival relative to that after treatment with doxorubicin. Herein, we demonstrate that the combination of Btz with the histone deacetylase (HDAC) inhibitor suberoylanilidehydroxamic acid (SAHA, also known as vorinostat) potently reactivates KSHV lytic replication and induces PEL cell death, resulting in significantly prolonged survival of PEL-bearing mice. Importantly, Btz blocked KSHV late lytic gene expression, terminally inhibiting the full lytic cascade and production of infectious virus in vivo. Btz treatment led to caspase activation and induced DNA damage, as evidenced by the accumulation of phosphorylated $\gamma \mathrm{H} 2 \mathrm{AX}$ and p53. The addition of SAHA to Btz treatment was synergistic, as SAHA induced early acetylation of p53 and reduced interaction with its negative regulator MDM2, augmenting the effects of Btz. The eradication of KSHVinfected PEL cells without increased viremia in mice provides a strong rationale for using the proteasome/ HDAC inhibitor combination therapy in PEL.
\end{abstract}

\section{Introduction}

Primary effusion lymphoma (PEL) is a rare, aggressive tumor caused by the Kaposi's sarcoma-associated herpesvirus (KSHV; also known as human herpesvirus-8 [HHV-8]) $(1,2)$. While PEL is a KSHV-driven tumor, coinfection with EBV is observed in approximately $80 \%$ of cases (3). PEL classically occurs in immunosuppressed individuals, most commonly in the setting of HIV infection, but may also develop in elderly patients $(4,5)$. PEL usually manifests as pleural, pericardial, or peritoneal effusions without contiguous tumor masses (5) and has dismal survival with conventional chemotherapy (6). The rarity of PEL and lack of suitable animal models have hindered the investigation of new therapeutic approaches that are urgently needed for this disease.

$\mathrm{KSHV}$ is an oncogenic $\gamma 2$-herpesvirus that pathogenically infects endothelial cells and B lymphocytes. It is implicated in the pathogenesis of PEL, Kaposi's sarcoma, multicentric Castleman's disease, and large B cell lymphoma arising from multicentric Castleman's disease $(2,5)$. The replicative cycle of KSHV exists as 2 canonical states: latency and lytic replication. Latency is the default state during which a restricted subset of viral transcripts is expressed. Lytic replication is required for KSHV production and propagation but may also contribute to the virus' oncogenic potential $(7,8)$. The lytic cycle occurs as a coordinated cascade of immediate early (IE), early, and late phases. IE genes transactivate and promote expression of early lytic genes, which are expressed

Authorship note: Shruti Bhatt and Brittany M. Ashlock are co-first authors. Enrique A. Mesri, Izidore S. Lossos, and Juan Carlos Ramos are co-senior authors. Conflict of interest: The authors have declared that no conflict of interest exists. Citation for this article: J Clin Invest. 2013;123(6):2616-2628. doi:10.1172/JCI64503. prior to viral DNA replication. The late lytic genes expressed after DNA replication allow mature virion formation and egress with subsequent cell death.

During latent infection, KSHV lytic gene expression is epigenetically repressed by chromatin condensation and histone deacetylation $(9,10)$. Therefore, the replicative state can be induced using histone acetyl transferase recruiters, such as phorbol esters, and histone deacetylase (HDAC) inhibitors (HDIs) $(9,10)$. HDACs are overexpressed in various cancers (11), and HDIs are effective antineoplastic drugs. Several mechanisms have been proposed for the antitumor effects of HDIs, including chromatin decondensation and expression of silenced cell cycle regulators and tumor suppressor genes (12). Recent studies have shown that the clinically available pan-HDI suberoylanilidehydroxamic acid (SAHA, also known as vorinostat) is a highly effective inducer of viral lytic replication (13). Therefore, the dual antineoplastic and lytic-inducing role of HDIs may be exploited in the treatment of PEL and other virus-related malignancies.

We recently established a direct xenograft model of PEL (UMPEL-1), in which freshly isolated PEL cells derived from the peritoneal cavity of a PEL-bearing patient were injected directly into NOD/SCID mice, resulting in progressive and reproducible tumor growth similar to the human PEL. In this model, we demonstrated that the 26S proteasome inhibitor, bortezomib (Btz), induced KSHV lytic gene expression in the primary PEL cells and increased survival of PEL-bearing NOD/SCID mice when compared with doxorubicin treatment (14). Indeed, Btz has emerged as an effective antineoplastic drug in several cancers and has clinical activity in PEL (14-16).

In this study, we hypothesized that targeting KSHV latency with the antineoplastic and lytic-inducing combination of Btz 
A
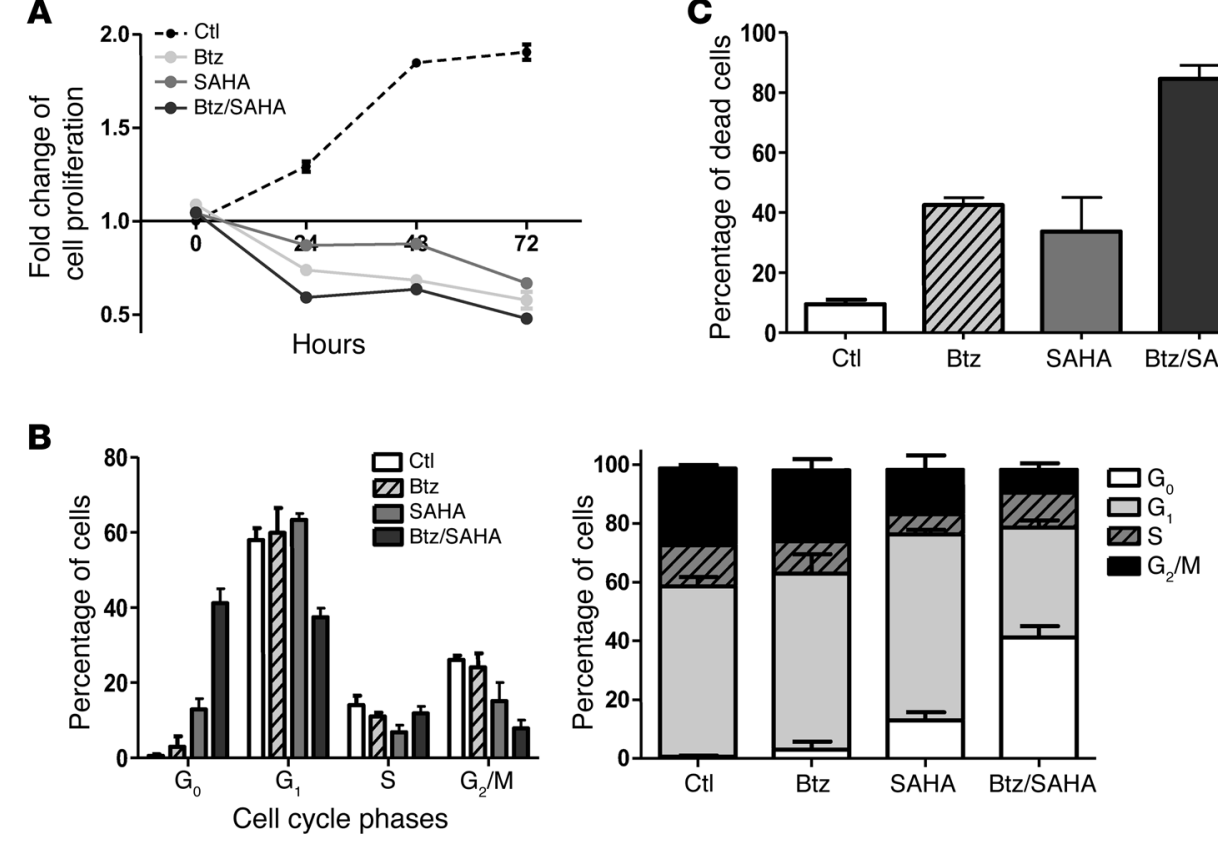

Figure 1

The combination of Btz with SAHA inhibits proliferation, induces cell cycle arrest, and triggers apoptosis of UM-PEL-1 cells. UM-PEL-1c cells were treated with $10 \mathrm{nM} \mathrm{Btz,} 0.75 \mu \mathrm{M}$ SAHA, or $10 \mathrm{nM} \mathrm{Btz} / 0.75 \mu \mathrm{M}$ SAHA. (A) The fold change in cell proliferation, as assessed by MTS assay at $0,24,48$, and 72 hours after treatment. (B) Analysis of the percentage of cells in different phases of the cell cycle $\left(G_{0}, G_{1}, S\right.$, and $\mathrm{G}_{2} / \mathrm{M}$ ) at 24 hours after treatment with the indicated drugs. Cells were stained with PI to measure DNA content and analyzed for cell cycle distribution by flow cytometry. ctl, control. (C) The percentage of dead cells measured by flow cytometric analysis of YO-PRO-1/PI-stained cells 24 hours after treatment. Experiments depicted in $\mathbf{A}-\mathbf{C}$ were repeated $\mathbf{3}$ times independently in triplicate. The representative data from 1 experiment is shown. Error bars represent SEM.

and SAHA (Btz/SAHA) would potently induce PEL cell death. We found that Btz/SAHA synergized to induce KSHV lytic replication and massive apoptosis, resulting in prolonged survival of PEL-bearing mice. Importantly, Btz also interfered with the full KSHV lytic replication, resulting in inhibition of infectious virus production, indicating that Btz functions as both inducer and inhibitor of KSHV replication in vivo. Further investigation into the antitumor mechanisms revealed that Btz led to caspase activation in PEL xenografts and induced DNA damage, as evidenced by phosphorylation of $\gamma$-histone 2AX $(\gamma \mathrm{H} 2 \mathrm{AX})$ and increased levels of phosphorylated p53. Further, SAHA induced early acetylation of $\mathrm{p} 53$ and promoted its dissociation from the negative regulator MDM2. Our study provides a strong rationale for the combined use of Btz and SAHA in PEL, an approach that may be clinically beneficial in immunocompromised patients suffering from $\gamma$-herpesvirus-induced malignancies.

\section{Results}

The Btz/SAHA combination blocks proliferation and induces cell cycle arrest and apoptosis in PEL cells. Given our previous observations that Btz induces KSHV lytic replication and confers a survival advantage in PEL-bearing mice, we tested the Btz/SAHA combination for PEL treatment. We hypothesized that if the outcome of KSHV lytic replication is apoptosis through a cytopathic effect, the combination of these drugs should induce greater apoptosis and thus confer a longer survival advantage for mice bearing PEL that Btz/SAHA induced higher levels of apoptosis in UM-PEL-1c, BC-1, and BC-3 cells compared with single drugs in a dose-dependent manner (Figure 1C and Supplemental Figure 1B). Btz and SAHA at $5 \mathrm{nM}$ and $0.5 \mu \mathrm{M}$, respectively, induced approximately $30 \%$ of apoptosis in BC1, BC3, and UM-PEL-1 cells, but the $5 \mathrm{nM}$ $\mathrm{Btz} / 0.5 \mu \mathrm{M}$ SAHA combination induced apoptosis in approximately $60 \%$ of the cells (Supplemental Figure 1C). By increasing the doses of Btz and SAHA to $10 \mathrm{nM}$ and $0.75 \mu \mathrm{M}$, respectively, the drug combination induced apoptosis in more than $80 \%$ of UM-PEL-1c cells (Figure 1C). Overall, these findings demonstrate that the combination of Btz/SAHA is more effective at inhibiting cell proliferation, inducing cell cycle arrest and apoptosis of PEL cells, compared with either drug alone.

The combination of Btz/SAHA synergistically induces KSHV lytic replication in PEL cells. To determine the effect of Btz/SAHA combination on KSHV lytic induction in UM-PEL-1c cells, genes representing all stages of the viral replicative cycle were analyzed by quantitative RT-PCR (qRT-PCR) at 24 hours after treatment. Compared with individual treatment with Btz or SAHA, the Btz/SAHA combination induced an additive or synergistic upregulation of the IE genes (ORF50, also known as RTA, and ORF45) and early genes (ORF21, also known as $v T K$; ORF36, also known as $v P K$ ) (Figure 2A). While both Btz and SAHA induced viral G protein-coupled receptor (ORF74, also known as vGPCR) expression at both the mRNA and protein levels, as measured by qRT-PCR and immunofluorescence assay, respectively, Btz/ 

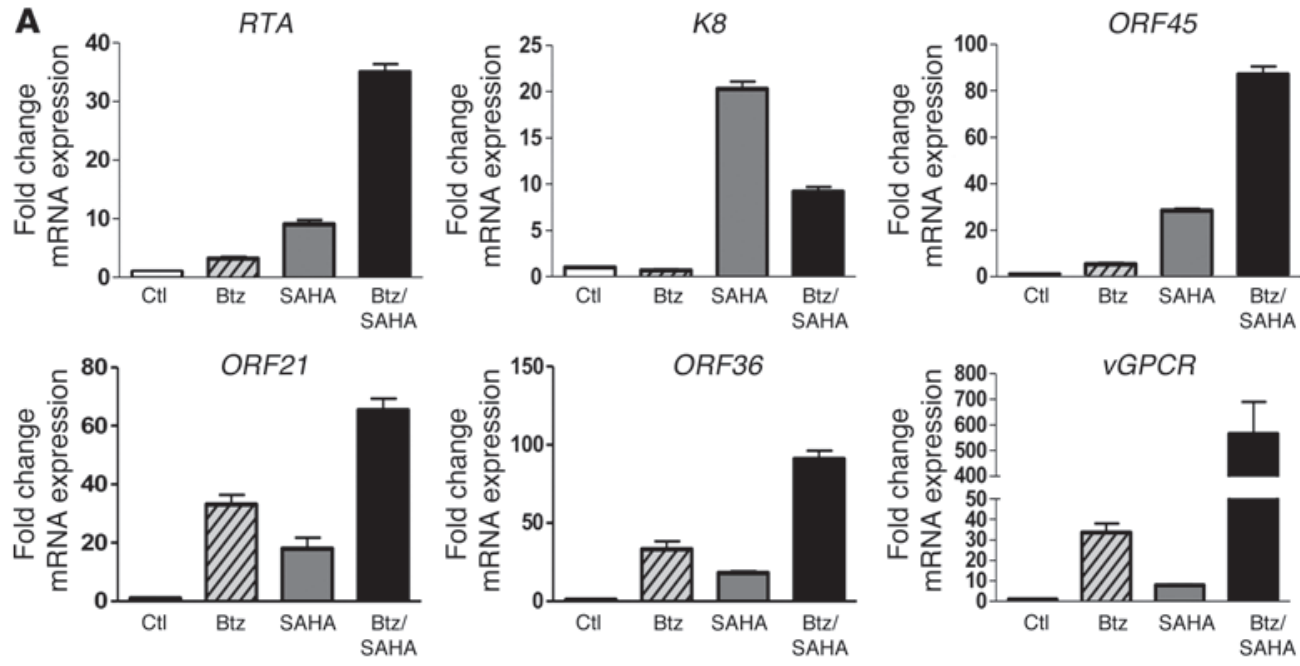

B
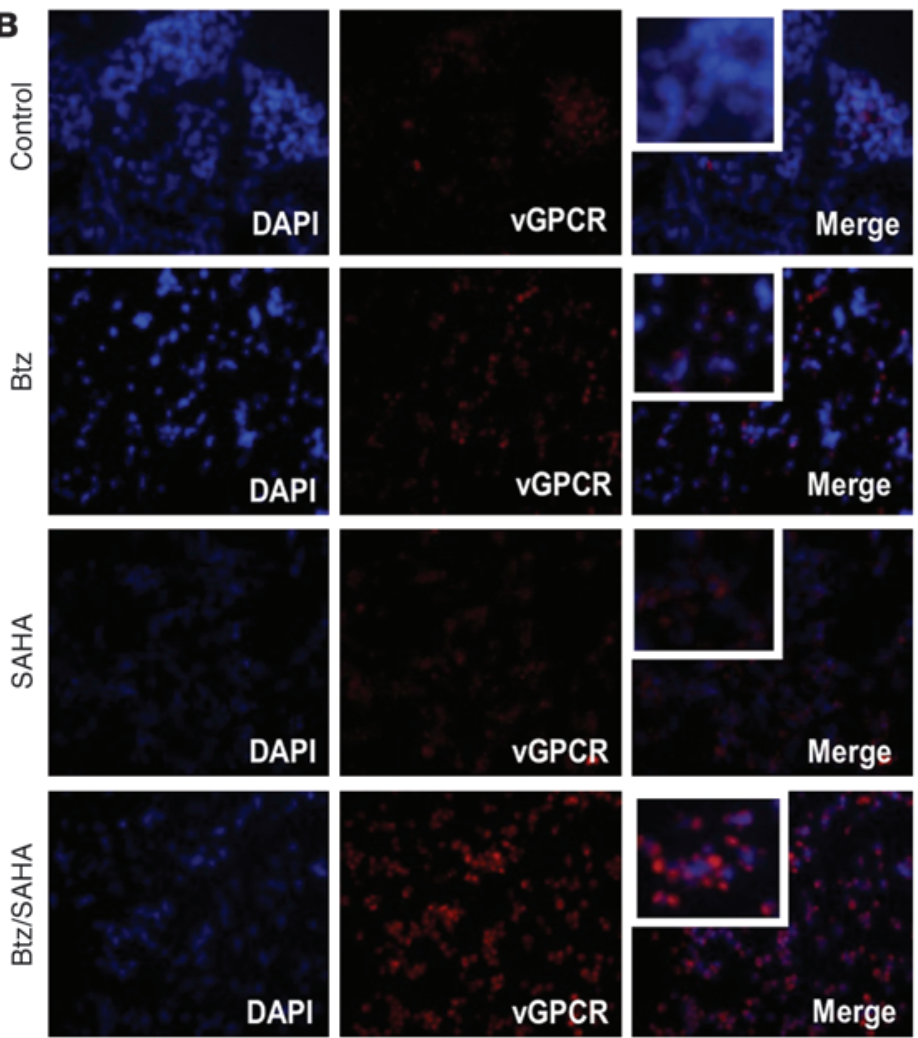

D

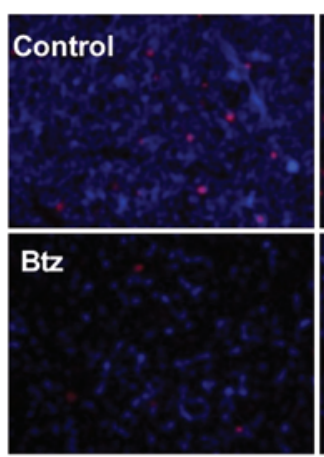

SAHA

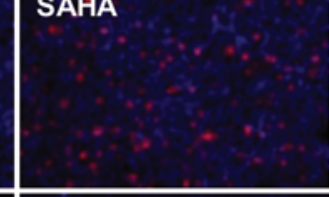

Btz/SAHA

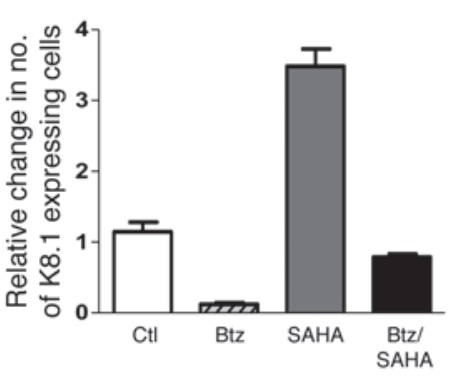

C

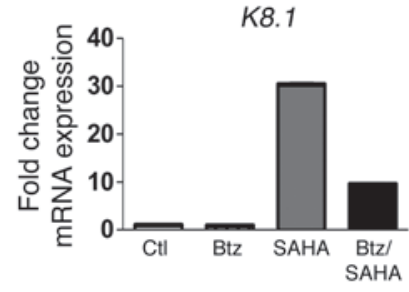

\section{Figure 2}

Btz and SAHA synergize to induce KSHV lytic replication, while concurrently inhibiting expression of selective lytic genes in UM-PEL-1c cells. UM-PEL-1c cells were treated with $10 \mathrm{nM} \mathrm{Btz,} 0.5 \mu \mathrm{M}$ SAHA, or $10 \mathrm{nM}$ Btz/0.5 $\mu \mathrm{M}$ SAHA for 24 hours. (A and C) Total RNA was harvested for qRTPCR analysis of viral mRNA expression. (B and D) Cells were cytospun and fixed, and immunofluorescence was performed for viral proteins. (A) qRT-PCR analysis reveals that Btz and SAHA induce expression of IE and early lytic viral mRNAs, with the exception of $K 8$, which was inhibited by Btz. (B) The vGPCR protein was immunostained to determine the extent of viral reactivation. Widespread reactivation is seen in the cells treated with the Btz/ SAHA combination. (C) K8.1, a late lytic viral gene, was inhibited by Btz at the transcript level. (D) Immunostaining images for K8.1 protein confirm the inhibition of K8.1 expression by Btz. K8.1 expression was normalized to DAPI to account for potential differences in cell number (left). Relative increase in K8.1-positive cells after treatment with SAHA alone and decrease in K8.1-positive cells after adding Btz was observed (right). Results are representative of 3 individual experiments. Error bars represent SEM. Original magnification, $\times 200$ (B); ×100 (D). 
A

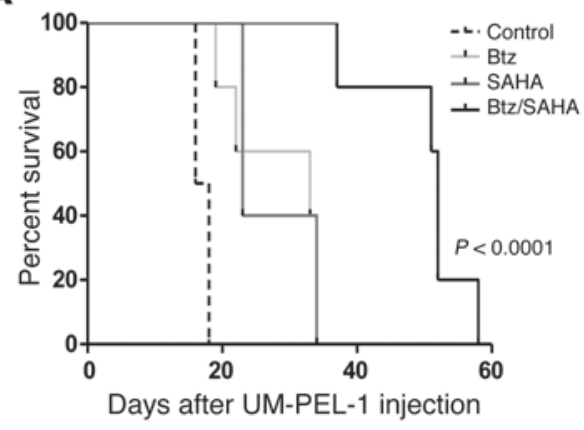

B

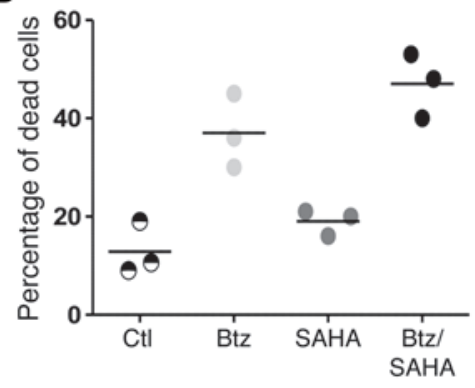

C
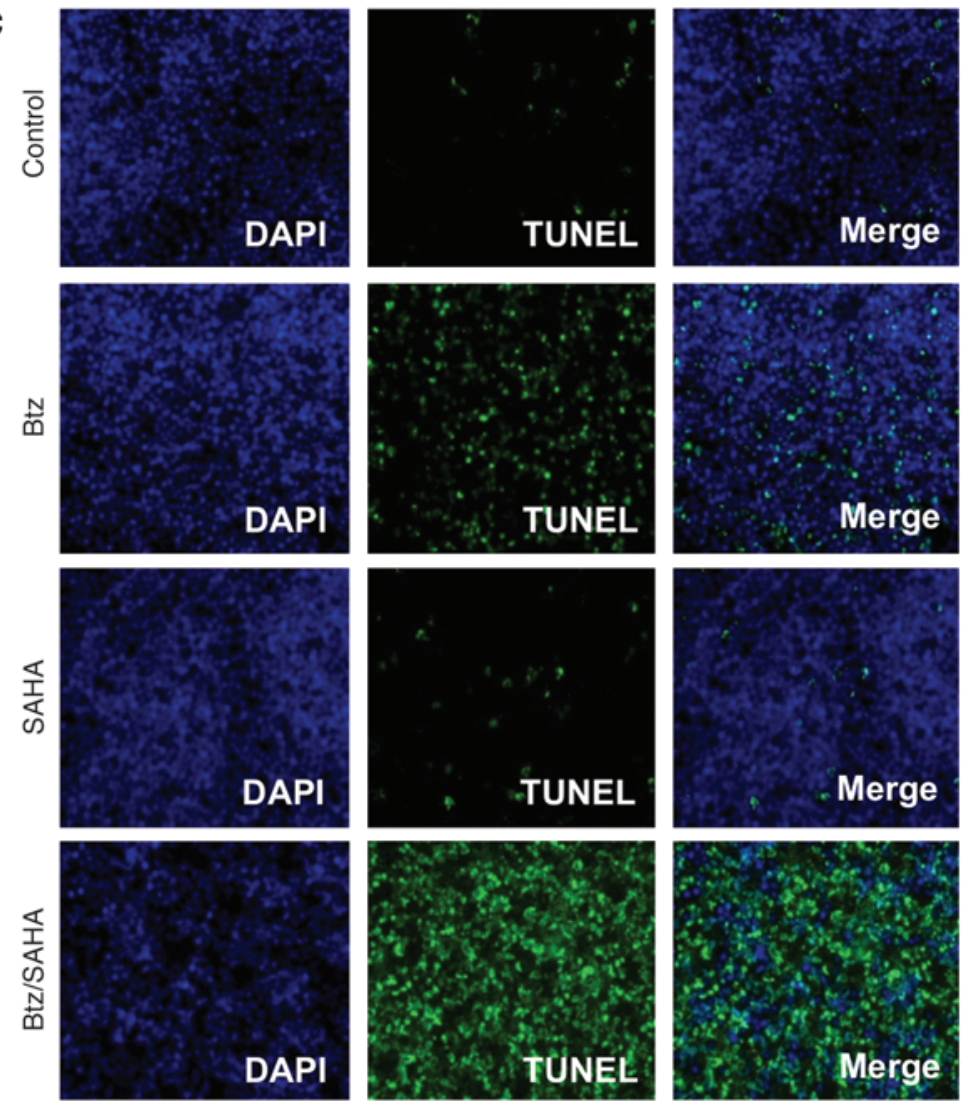

D

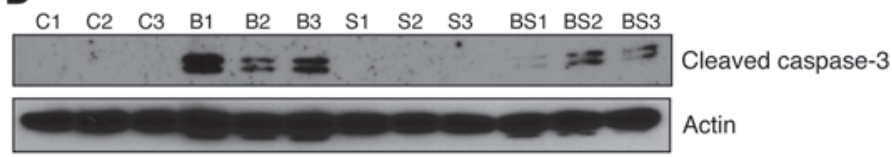

E

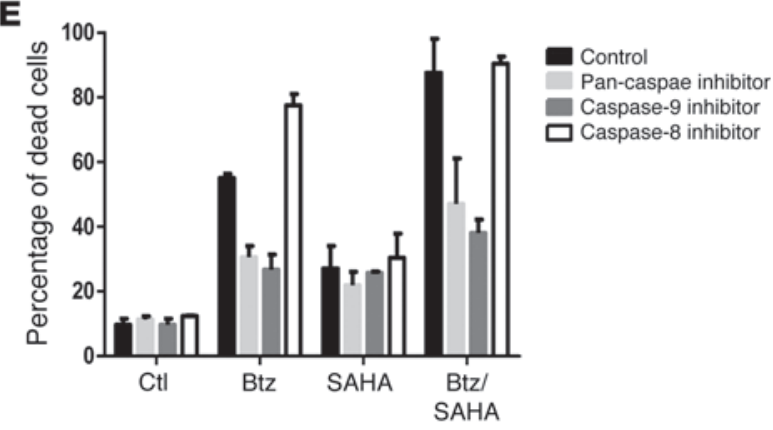

Figure 3

Btz and SAHA combination prolongs survival of PEL-bearing mice by inducing apoptosis in PEL xenografts. (A) Kaplan-Meier survival curve of NOD/SCID mice ( $n=5$ mice per group) injected i.p. with UM-PEL-1 cells and treated with indicated drug combinations, as described in Methods. Results are representative of 2 independent experiments. (B-D) UM-PEL-1-bearing mice $(n=3)$ were treated separately with a single dose of DMSO (control [Ctt]), Btz, SAHA, or Btz/SAHA. At 24 hours, the mice were sacrificed and UM-PEL-1 cells were harvested. (B) In vivo apoptosis of UM-PEL-1 cells examined by YO-PRO-1/PI staining followed by flow cytometry. Each circle represents 1 mouse, and the horizontal bars indicate the mean percentage apoptosis. (C) Immunofluorescence images of TUNEL-stained (green, middle column) UM-PEL-1 cells harvested from peritoneal effusions. Nuclei are stained with DAPI (blue, left column). Merged images of DAPI and TUNEL are shown in the right column. TUNEL positivity is indicative of fragmented DNA, a characteristic of apoptotic cells. Original magnification, $\times 100$. (D) Immunoblotting for cleaved caspase-3. $\beta$-Actin was used as normalizing control. C, DMSO control; B, Btz; S, SAHA, BS, Btz/SAHA. (E) UM-PEL-1c cells were pretreated with pan-caspase (Z-VAD-FMK), caspase-8 (Z-IETD-FMK; effector of extrinsic pathway), and caspase-9 (Z-LEHD-FMK; effector of intrinsic pathway) specific inhibitors $(50 \mu \mathrm{M})$ for 2 hours, followed by treatment with $2.5 \mathrm{nM} \mathrm{Btz}, 0.5 \mu \mathrm{M}$ SAHA, or $2.5 \mathrm{nM}$ Btz/0.5 $\mu \mathrm{M}$ SAHA for 24 hours. The percentage of dead cells was measured by flow cytometric analysis of YO-PRO-1/PI-stained cells. Ctl, control cells not treated with Btz or SAHA. Error bars represent SEM between triplicate samples.

SAHA was more effective at upregulating this early lytic viral gene (Figure 2, A and B). Moreover, vGPCR staining indicated a very high ratio of lytic reactivation for the SAHA/Btz combination that correlates with its apoptotic activity, as depicted in Figure 1. The late lytic gene K8.1 was 30 -fold induced in SAHA- only-treated cells but remained unaffected in the Btz-treated cells (Figure 2C). Furthermore, concurrent exposure to Btz abrogated the SAHA-induced K8.1 upregulation by approximately $60 \%$ (Figure 2C). This K8.1 transcriptional pattern was mimicked at the protein level with a decrease in the percentage of K8.1-expressing 

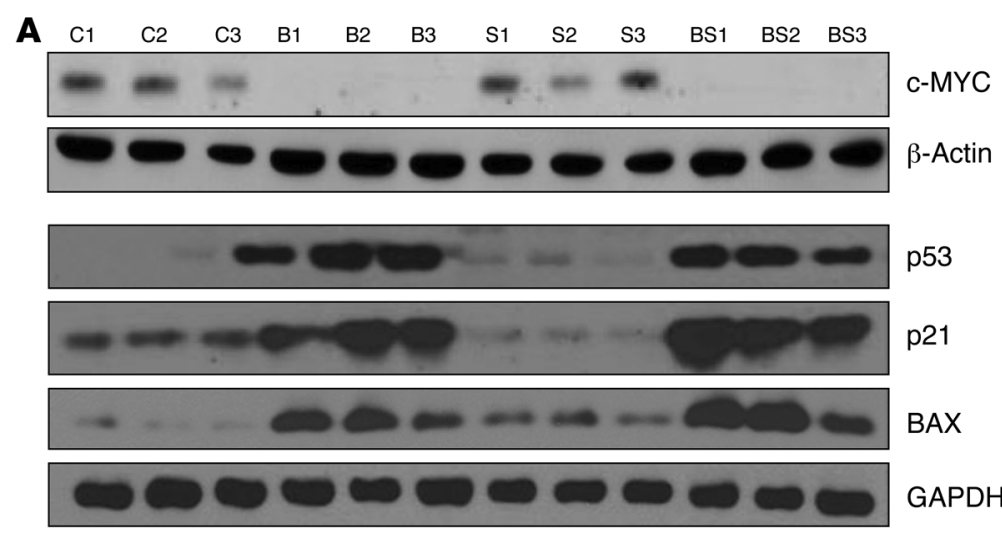

p53

p21

BAX

GAPDH

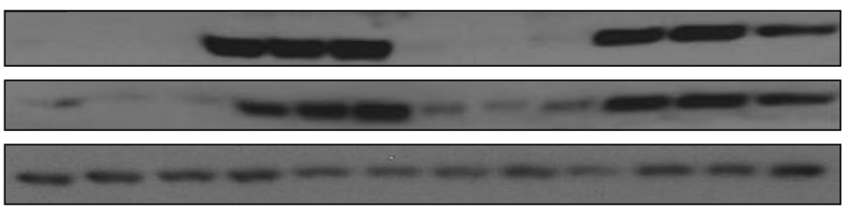

$\mathrm{pH} 2 \mathrm{AX}$

H2AX

GAPDH
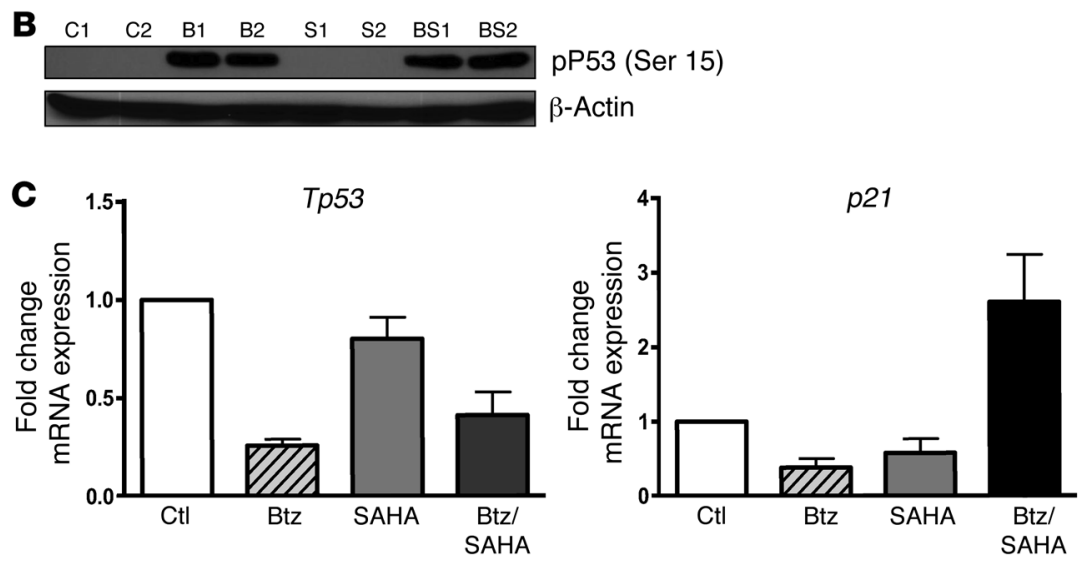
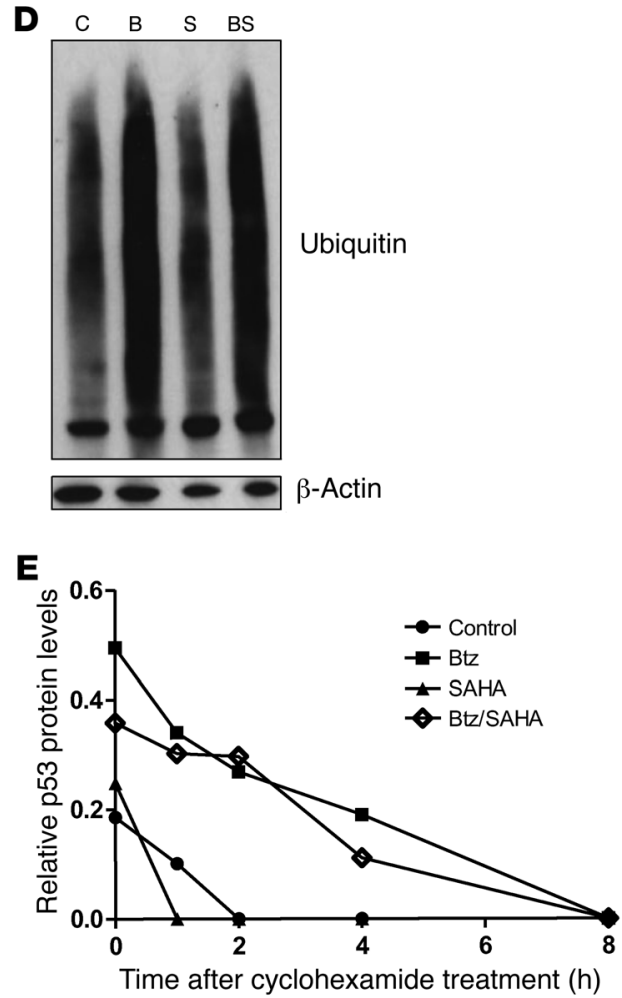

\section{Figure 4}

Btz downregulates c-MYC and stabilizes the expression of phosphorylated p53 and $\gamma \mathrm{H} 2 \mathrm{AX}$ and downstream p53 targets p21 and Bax in PEL xenografts. (A) UM-PEL-1-bearing mice ( $n=3$ mice per group) were treated with DMSO as control or a single dose of Btz (0.3 mg/kg), SAHA (60 $\mathrm{mg} / \mathrm{kg}$ ), or the combination (Btz/SAHA) for 2 or 24 hours. UM-PEL-1 cells harvested from mouse ascites, and whole cell lysates were prepared for immunoblotting using the indicated antibodies. (B) UM-PEL-1-bearing mice ( $n=2$ mice per group) were treated as above, and immunoblotting for Ser-15-phosphorylated p53 (P-p53) was performed. (C) Fold change in p53 (Tp53) and p21 mRNA levels was measured using qRT-PCR analysis. Error bars indicate standard deviation between triplicate samples. Data are representative of 2 independent experiments. Ctl, DMSO-treated control. (D) Immunoblotting for Lys-48 polyubiquitin using UM-PEL-1 whole lysates following 24 hours treatment in vivo. (E) UM-PEL-1-bearing mice were treated with a single dose (as indicated in A) of Btz, SAHA, and the Btz/SAHA combination. At 24 hours after treatment, UM-PEL-1 cells were harvested and exposed to cycloheximide $(50 \mu \mathrm{M})$ in culture. At the indicated time periods (ranging from 0 to 8 hours), p53 protein levels were measured by immunoblotting and quantified by densitometry. The half-life of p53 was determined by plotting p53 levels normalized to GAPDH ( $y$ axis) and time after treatment with cycloheximide ( $x$ axis). Note the higher starting amounts of p53 in the Btz and Btz/SAHA treatment groups.

cells following Btz treatment (Figure 2D). This effect appeared to be locus-specific, since the IE gene $K 8$ exhibited a similar pattern of mRNA expression as that of K8.1 (Figure 2A). Other KSHV genes (ORF8, also known as $g B$; ORF49; and ORF73, also known as $L A N A$ promoter) were slightly induced in Btz-treated cells and were induced more with SAHA treatment, but were synergistically activated with the combination treatment, while Kaposin was strongly induced by Btz, regardless of SAHA treatment, indicating that Btz differentially affects KSHV gene expression (see below and Supplemental Figure 2). Overall our data indicate that Btz and SAHA synergize to induce KSHV lytic replication with selec- tive repression of some IE and late lytic genes correlating with the high rates of PEL cell cytotoxicity.

The combination of Btz/SAHA induces marked apoptosis in PEL xenografts and enhances survival of tumor-bearing NOD/SCID mice. The in vitro antiproliferative and cytotoxic activities of Btz/SAHA in multiple PEL cell lines suggested therapeutic potential in vivo. Therefore, we evaluated the effects of Btz/SAHA in a direct xenograft UM-PEL-1 model, originally established directly from a patient with PEL and continuously propagated in NOD/SCID mice, allowing for the maintenance of the original PEL phenotype (14). Four groups of NOD/SCID mice $(n=5)$ were inocu- 
A

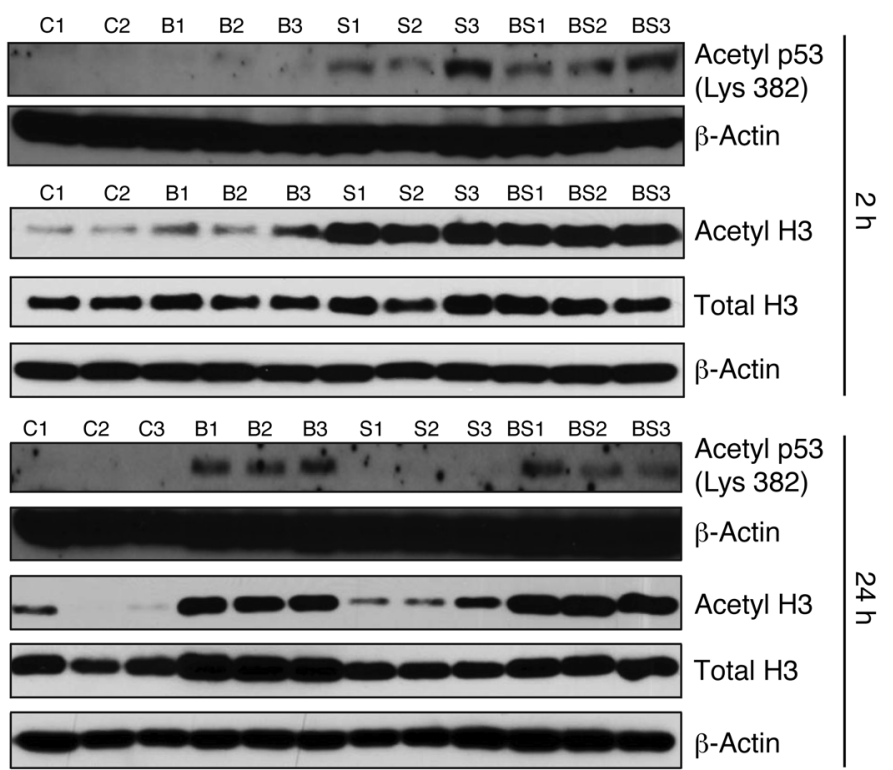

B

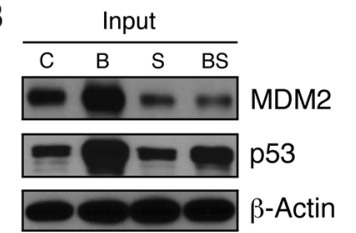

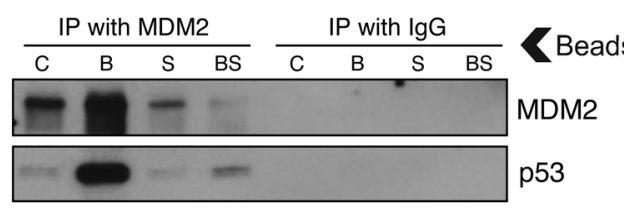

$\frac{\text { IP with MDM2 }}{\text { C B S BS Flow-through }}$
$-\infty \quad-\quad$ D53
C

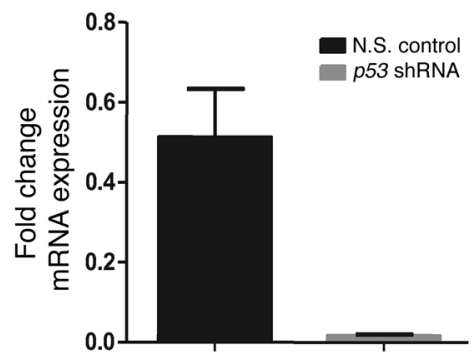

D

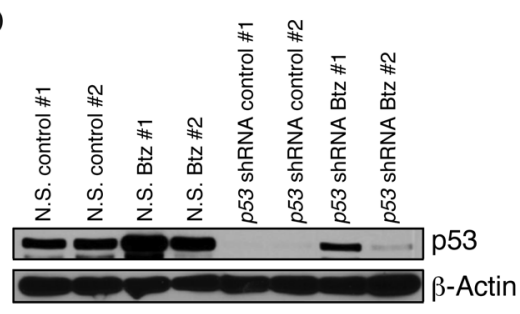

E
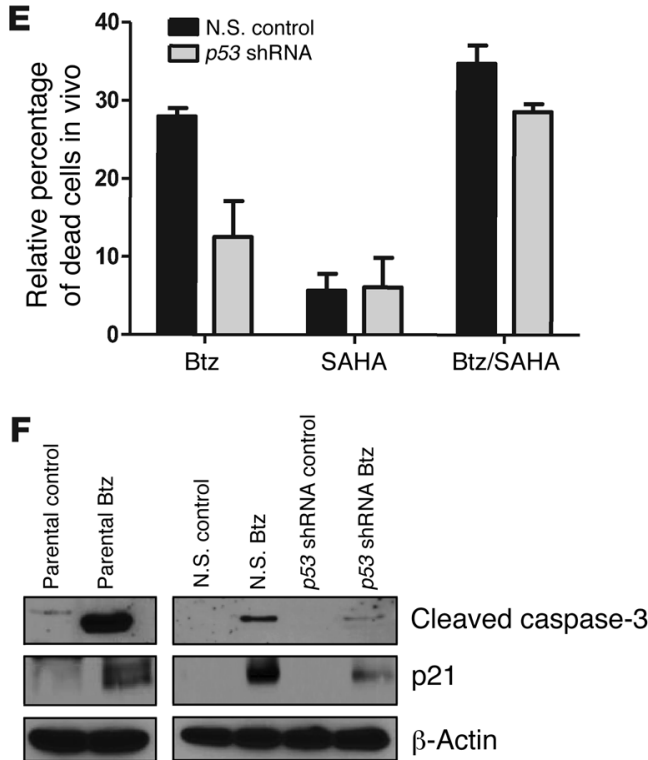

G

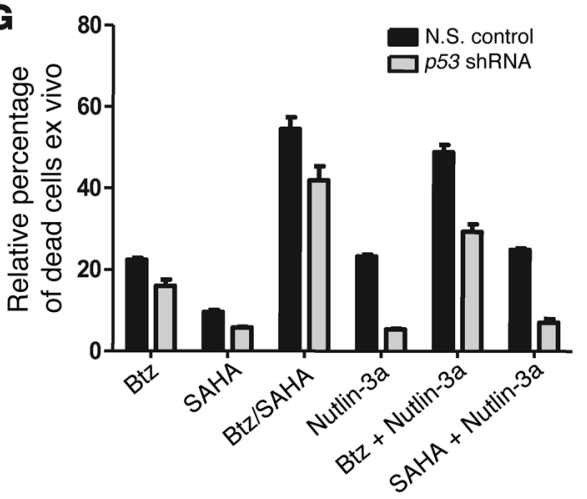

Figure 5

SAHA-induced acetylation of p53 led to dissociation from MDM2, contributing to cell apoptosis. (A and B) Tumor-bearing mice were treated with a single dose of DMSO, SAHA, Btz, or Btz/SAHA, and cell protein lysates were prepared from peritoneal effusions. (A) Immunoblotting for acetylated p53 (Lys-382) and H3 at 2 hours and 24 hours after treatment. (B) Immunoblots for MDM2 and p53 in whole cell lysates prior to immunoprecipitation (left). Immunoblots showing MDM2 and p53 in complexes captured by immunoprecipitation using indicated antibodies (right top). Complex-free p53 in flow through supernatant not captured in MDM2 antibody resin bead complexes (right bottom). (C-F) UM-PEL-1 cells stably expressing p53-specific shRNA or nonsilencing (N.S.) vectors were passaged as xenografts in mice. Mice ( $n=2$ per group) were treated with a single dose of DMSO, Btz, SAHA, or Btz/SAHA, and at 24 hours, cells were harvested from peritoneal effusions. (C and D) shRNA-mediated knockdown of $p 53$ at the mRNA level in untreated UM-PEL-1 cells and partial abrogation of Btz-induced p53 levels measured by qRT-PCR and immunoblotting. (E) In vivo apoptosis of UM-PEL-1 cells examined by YO-PRO-1/PI staining, followed by flow cytometry. Data represent values relative to normalized DMSO-treated control mice. (F) Immunoblotting of p21 and activated caspase-3. (G) UM-PEL-1 cells harvested from p53 shRNA- or nonsilencing vector-expressing xenograft mice were treated in culture with $2.5 \mathrm{nM} \mathrm{Btz,} 0.5 \mu \mathrm{M} \mathrm{SAHA}, 7 \mu \mathrm{M}$ nutlin-3a, or indicated combinations for 24 hours. The percentage of dead cells was measured by annexin V/PI staining. Data represent values relative to normalized untreated cells. (A-C) Experiments were repeated twice in triplicates. Error bars represent SEM. 
A
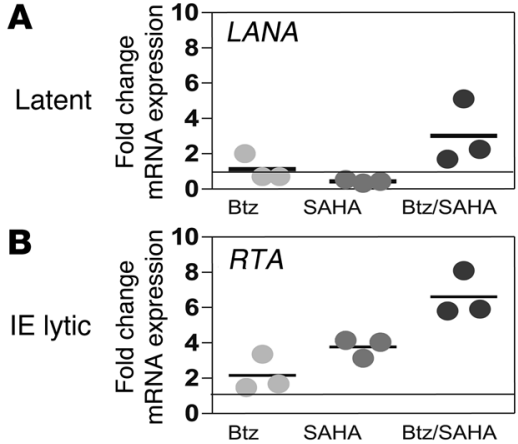

C

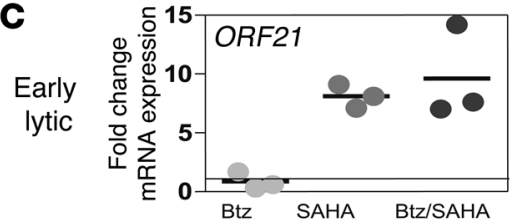

D

Late

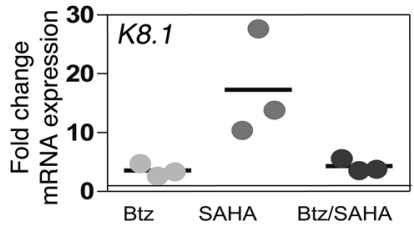

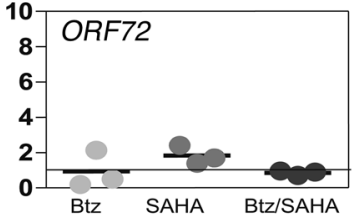
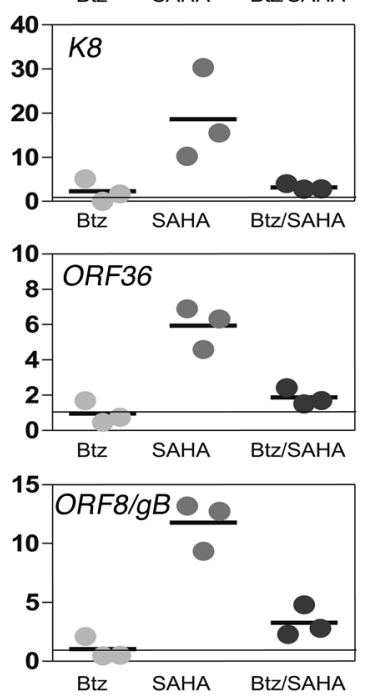
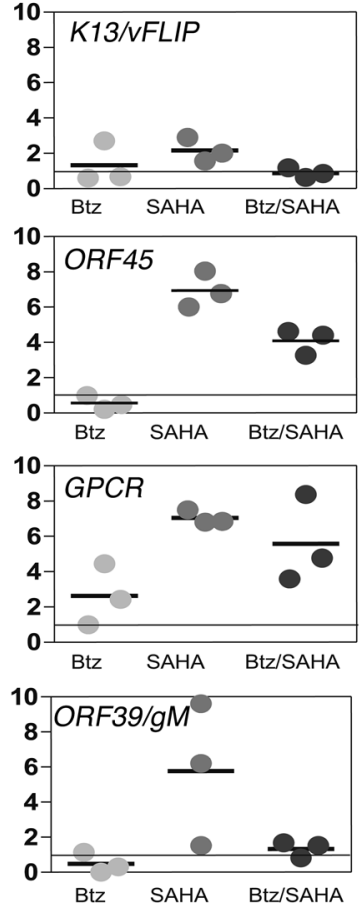

$\mathbf{E}$

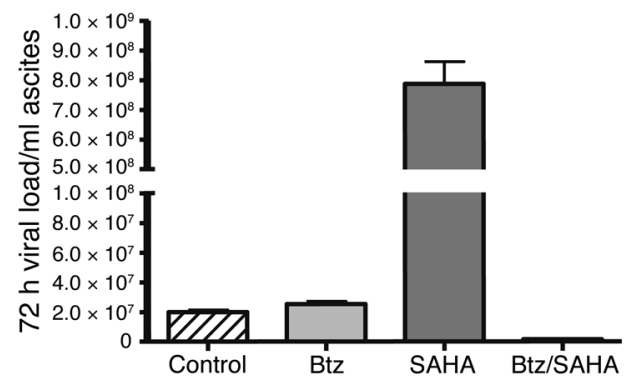

lated i.p. with UM-PEL-1 cells and treated i.p. with Btz (0.3 mg/ $\mathrm{kg}$ twice weekly), SAHA (60 mg/kg daily), Btz/SAHA combination, or DMSO (50 $\mu$ l daily) for 3 weeks, starting on day 3 after tumor inoculation when all mice had visible abdominal distension secondary to malignant ascites; all regimens were well tolerated. To confirm the ascites was due to the growth of UM-PEL-1 cells, peritoneal cells collected from lymphoma-bearing mice on day 7 were 97.23\% positive for CD30 (Supplemental Figure 3), suggesting that majority of cells in the ascites are indeed UM-PEL-1. When left untreated, these mice died within approximately 15 days. Treatment with SAHA alone showed comparable efficacy to that of Btz, extending the overall survival compared with that of control mice (median survival of 23, 33, and 15 days for SAHA, Btz, and DMSO control, respectively; Figure 3A). However, the Btz/SAHA combination led to a further increase in the overall survival as compared with that of single-drug or DMSO control groups (median survival of 57 days; $P<0.0001$; Figure 3A). There was increased apoptosis of UM-PEL-1 cells obtained from mice treated for 24 hours with single dose of the Btz/SAHA combination ( $47 \% \pm 3.78 \%$ ) and Btz $(37 \% \pm 4.35 \%)$ compared with that of those from mice treated with SAHA $(19 \% \pm 1.52 \%)$ and DMSO control $(12.86 \% \pm 3.1 \%)$, as assessed by YO-PRO-1/PI staining (Figure 3B). This was further confirmed by TUNEL assay, which demonstrated that most cells

in the combination-treated mice exhibited signs of DNA fragmentation and were committed to apoptosis (Figure 3C).

Btz-induced apoptosis in PEL is mediated via the intrinsic mitochondrial pathway but not through the unfolded protein response or NF-KB inhibition. The mechanism of Btz-induced apoptosis in PEL remains unclear. After confirming apoptotic potential of Btz/SAHA combination in PEL cell lines and UM-PEL-1 xenografts, we next investigated the potential mechanism(s) of apoptosis. Activation of caspase cascade is a pivotal step in apoptosis. Caspases can be activated via extrinsic (death receptor-mediated) or intrinsic (mitochondriamediated) pathways. Btz and Btz/SAHA treatment in UM-PEL-1 xenografts led to caspase activation, as demonstrated by caspase-3 cleavage (Figure 3D). To investigate whether this was due to activation of intrinsic versus extrinsic pathways, UM-PEL-1 cells were treated ex vivo with several caspase inhibitors. Treatment of UM-PEL-1 cells with ZVAD-FMK (pan-caspase) and caspase-9-specific inhibitor (effector of intrinsic pathway) resulted in a marked decrease in Btz- and Btz/SAHA-induced apoptosis as compared with that in controls (Figure $3 \mathrm{E}$ ). By contrast, inhibition of caspase-8 (effector of extrinsic pathway) failed to prevent cell death induced by Btz treatment. Overall these studies suggest that Btz-induced apoptosis is mediated through the activation of the intrinsic pathway in PEL. 

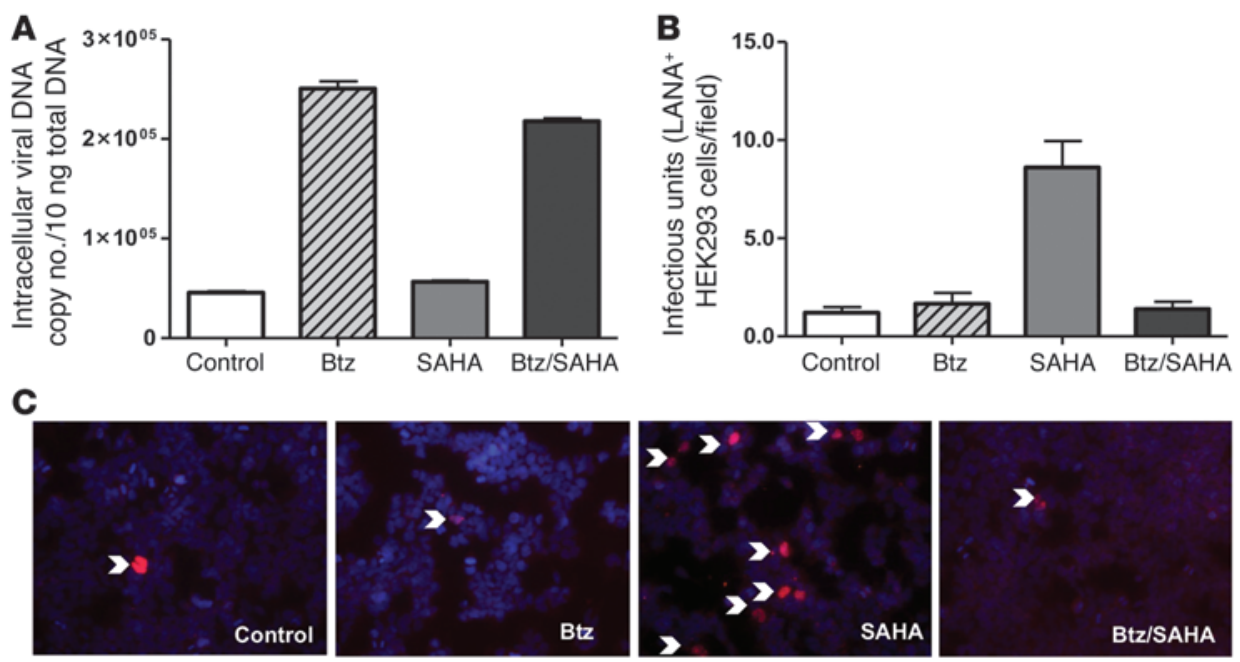

D

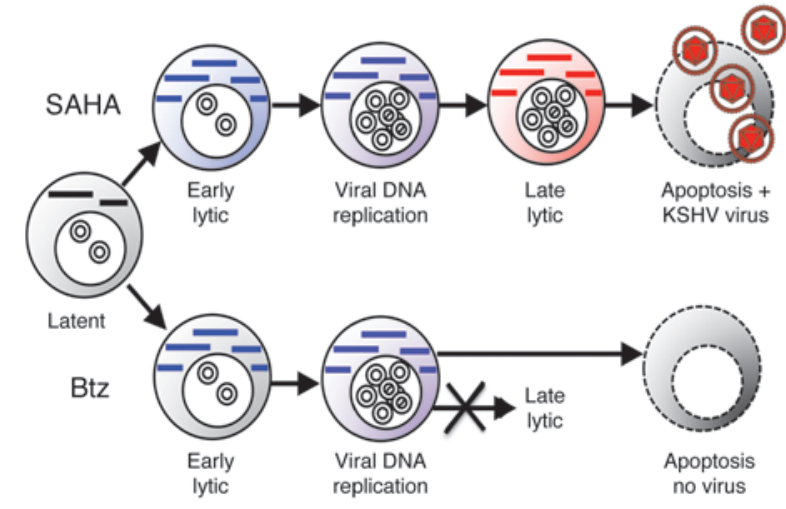

\section{Figure 7}

Btz induces accumulation of viral DNA, with concomitant inhibition of infectious virion production. (A) Cultured UM-PEL-1c cells were treated for 72 hours with $10 \mathrm{nM} \mathrm{Btz,} 0.5 \mu \mathrm{M}$ SAHA,

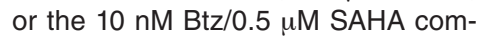
bination. Total intracellular DNA was isolated, and $10 \mathrm{ng}$ was used for GPCR viral load determination. (B and $\mathbf{C}$ ) Cell-free supernatant from PBS-treated (Control-treated), Btz-treated, SAHAtreated, or Btz/SAHA-treated cells was applied to uninfected HEK293 cells. Forty-eight hours later, the cells were fixed and stained for LANA. For graphing purposes, 1 non-doublet LANA-positive 293 cell was defined as 1 infectious unit. Immunofluorescence of a representative field for each condition is shown. Results are representative of 2 independent experiments. Error bars represent SEM. Original magnification, $\times 200$. (D) Mechanism of action for SAHA, Btz, and the combination. SAHA induces the full KSHV lytic cycle, leading to virus production and apoptosis. Btz also induces the lytic cycle, but it also blocks late lytic gene expression, leading to apoptosis in the absence of virus production. In the combination, SAHA and Btz synergize to induce the lytic cycle; however, the presence of Btz inhibits the completion of the lytic cycle, resulting in massive apoptosis in the absence of virus production.
Prior studies have demonstrated that Btz-mediated cell killing can occur through the induction of ER stress from the accumulation of nondegraded proteins, leading to the activation of the unfolded protein response (UPR) (17). Our previous study demonstrated that Btz had no significant effect on the UPR in UM-PEL-1c cells in vitro (14). To evaluate the effect of Btz on the UPR in UM-PEL-1 xenografts in vivo, we measured the expression of proteins known to be upregulated by the UPR (CHOP, GRP-78, GRP-94, ATF6, total and phosphorylated eIF2 $\alpha$ ) by immunoblotting. Btz, alone and in combination with SAHA, led to increased CHOP expression; however, no significant changes in the expression of other proteins, including phospho-eIF2 $\alpha$, were observed (Supplemental Figure 4A). qRT-PCR analysis of spliced $\mathrm{XBP} 1(s p X b p 1)$, which is normally induced upon UPR activation, revealed no change in its expression in the Btz-treated mice, whereas treatment with SAHA alone or in combination with Btz reduced the spXbp1 mRNA levels (Supplemental Figure 4B).

Other investigators have shown that Btz inhibits NF- $\mathrm{B}$ function inducing apoptosis in PEL cell lines (18). Constitutive NF-кB activity is a critical prosurvival mechanism for PEL, and inhibiting NF-кB function induces PEL cell apoptosis $(19,20)$. Therefore, we analyzed the effect of Btz/SAHA on NF- $\kappa \mathrm{B}$ activity in vivo using nuclear extracts from PEL xenografts by EMSA. Contrary to our previous study, in which we observed that Btz had no significant effect on NF-KB activity on UM-PEL-1 cells treated in vitro (14), in this study, we found that Btz induced IкB- $\alpha$ phosphorylation and increased NF- $\mathrm{KB}$ (p50/p65) DNA binding at 24 hours as com- pared with control (Supplemental Figure 5, A and B). Btz-mediated NF- $\kappa \mathrm{B}$ activation was further augmented in the presence of SAHA (Supplemental Figure 5, A and B). However, several NF-кB target genes, including cellular inhibitor of apoptosis protein (cIAP2), interferon regulatory factor 4 (Irf4), Bcl-2, and Bcl-XL remained unaltered upon treatment with Btz/SAHA, while cellular FLICE inhibitory protein (c-Flip) was induced (Supplemental Figure 5C). Overall, these observations suggest that inhibition of NF- $\mathrm{KB}$ signaling is not contributing to the mechanisms of Btz/ SAHA-induced PEL death.

Btz-induced apoptosis in PEL in vivo is accompanied by downregulation of c-MYC and the accumulation of phosphorylated $\gamma \mathrm{H} 2 \mathrm{AX}$ and $\mathrm{p} 53$, indicative of DNA damage. We previously demonstrated that Btz treatment of UM-PEL-1 xenografts resulted in downregulation of c-MYC target genes (14). c-MYC is an oncogenic protein that plays an important role in lymphomagenesis and is deregulated in PEL (21). c-MYC is also known to be stabilized by KSHV LANA (22). We observed that Btz dramatically downregulated c-MYC in PEL cells in vivo at both mRNA and protein levels (Figure 4A and Supplemental Figure 6). These results support our previous conclusion that Btz may contribute to UM-PEL-1 cell death via downregulation of c-MYC.

To further investigate the antitumor mechanisms of Btz/SAHA in UM-PEL-1 cells, we examined the expression of the tumor suppressor protein p53, which is inactivated by KSHV LANA (23). We observed marked stabilization of p53, concomitant with upregulation of its targets, p21 and Bax proteins, in the Btz- and 
Btz/SAHA-treated mice (Figure 4A), while SAHA alone led to only a slight increase in Bax levels. Next, we examined the phosphorylation status of p53. Phosphorylation of p53 at serine 15 (Ser-15) is a key event for its transactivating function (24). DNA damage results in the phosphorylation of $\mathrm{p} 53$ at Ser-15 and Ser-20, leading to a reduced interaction with its negative regulator MDM2 (25). In PEL xenografts, Btz treatment resulted in the accumulation of Ser-15-phosphorylated p53 (Figure 4B). The increased expression of p53 in vivo exclusively occurred at the protein level, while its mRNA levels were decreased (Figure 4C), suggesting that Btzmediated inhibition of the $26 \mathrm{~S}$ proteasome resulted in the accumulation of $\mathrm{p} 53$ protein. Consistent with this, Btz treatment also led to accumulation of Lys-48 polyubiquitinated proteins, which are normally targeted for proteasomal degradation (Figure 4D). By contrast, Btz/SAHA combination augmented p21 at both mRNA and protein levels, indicative of increased p53 function (Figure 4C and refs. 26,27). Neither Btz nor SAHA affected the expression of other important cell cycle regulators, including $\mathrm{p} 16, \mathrm{p} 27$, and $\mathrm{Rb}$ proteins (Supplemental Figure 7).

Since p53 activation is induced upon DNA damage, we examined the levels of $\gamma \mathrm{H} 2 \mathrm{AX}$, which becomes phosphorylated as a result of DNA double-strand breaks. Our results demonstrate increased levels of phosphorylated $\gamma \mathrm{H} 2 \mathrm{AX}$, indicative of Btzinduced DNA damage in PEL cells in vivo (Figure 4A).

According to previous studies, rises in p53 protein levels after DNA damage are due to its increased stability and half-life (28). To examine the half-life of p53 in PEL xenografts, UM-PEL-1bearing mice were treated with a single dose of Btz, SAHA, or Btz/ SAHA. Cells were harvested 24 hours after treatment, exposed to $50 \mu \mathrm{M}$ cycloheximide, and cultured for up to 8 hours. Compared with control and SAHA alone, both Btz- and Btz/SAHA-treated PEL xenografts exhibited a 4-fold increase in p53 half-life (Figure $4 \mathrm{E})$. Our results demonstrate that Btz induced the stabilization of phosphorylated p53 in vivo and increased $\gamma \mathrm{H} 2 \mathrm{AX}$ phosphorylation, suggesting that Btz causes DNA damage in PEL tumors.

Btz and SAHA lead to the accumulation of acetylated $p 53$ and histone proteins in PEL in vivo. SAHA is a hydroxamic acid that reversibly inhibits class I and class II HDACs, resulting in the acetylation of histones and other cellular proteins (29). Recent reports indicate that proteasome inhibitors can also increase acetylation of histones that may contribute to tumor cell apoptosis (30-32). HDAC1 is involved in p53 deacetylation on lysine residues by interacting with the p53-MDM2 complex (33). MDM2 forms a complex with p53 and catalyzes its ubiquitination on lysine residues, thus targeting p53 for rapid proteasomal degradation (34). Accordingly, we analyzed the effect of Btz and SAHA on histone and p53 acetylation at lysine 382 (Lys-382) by immunoblotting in PEL xenografts. As expected, SAHA caused increased levels of acetylated p53 and histone subunit 3 (H3) as early as 2 hours after treatment (Figure 5A). This effect was transient and reversible, since the SAHA-induced acetylation of both p53 and H3 diminished by 24 hours after treatment (Figure 5A). Noticeably, Btz also increased levels of acetylated p53 and $\mathrm{H} 3$ progressively from 2 to 24 hours after treatment. In cultured UM-PEL-1 cells, Btz/SAHA increased acetylated histone levels to a much greater extent than either drug alone (Supplemental Figure 8).

Next, as p53 acetylation at several lysine residue sites affects its function, including binding to its negative regulator MDM2 (35), we investigated the interaction between $\mathrm{p} 53$ and MDM2 proteins by immunoprecipitation 24 hours after Btz and SAHA treatment in vivo. We found reduced MDM2 protein levels and near complete disappearance of p53-MDM2 complexes in PEL xenografts treated with the Btz/SAHA combination as compared with that in those treated with Btz alone. By contrast, total MDM2-free $\mathrm{p} 53$ protein remained high in the xenografts treated with the Btz/SAHA combination (Figure 5B). The observed loss of p53-MDM2 complexes was likely not due to decrease in $M d m 2$ transcription, as only a partial reduction of mRNA was observed in the Btz/SAHA combination (Supplemental Figure 6). The stabilization of activated free p53 observed in Btz/SAHA-treated tumors correlated with an increase in p53 transcriptional activity, as evidenced by $\geq 3$-fold induction of $p 21$ mRNA levels as compared with those treated with control or either drug alone (Figure 4C). Taken together, these results suggest that the cooperative antineoplastic efficacy of Btz/ SAHA in PEL might be partly explained by (a) the ability of Btz to induce DNA damage and stabilize activated - phosphorylated $\mathrm{p} 53$, and (b) early hyperacetylation of p53 by SAHA, thus favoring the disruption of p53-MDM2 complexes, leading to MDM2 destabilization and increased overall p53 activity.

Btz-and Btz/SAHA-induced apoptosis in PEL is partially dependent on $p 53$. Our results showing a possible role of p53 in Btz/SAHAinduced apoptosis are consistent with results from recent studies, demonstrating that nutlin-3 induces apoptosis of PEL cells by disruption of $\mathrm{p} 53-\mathrm{MDM} 2$ interaction (36). Therefore, to determine a causative role of p53 in Btz/SAHA-induced apoptosis, we tested the ability of p53 knockdown to prevent apoptosis of UM-PEL-1 cells. UM-PEL-1 cells were transduced ex vivo with a lentivirus vector encoding $p 53$-specific shRNAs ( $p 53$ knockdown) or nonsilencing vector (mock) and passaged successfully as stable xenografts in mice. A highly efficient p53 knockdown was achieved at both mRNA and protein levels, as evidenced by a marked reduction of stabilized p53 after Btz treatment in vivo upon immediate culture (Figure 5, C and D). The $p 53$ knockdown partially prevented Btz-induced apoptosis, caspase- 3 cleavage, and p21 expression as compared with mock (Figure 5, E and F). To this end, we examined the apoptotic effects of Btz, SAHA, and nutlin-3 in $p 53$ knockdown cells versus mock UM-PEL-1 cells treated upon immediate culture. As expected, Btz and nutlin-3, alone or in combination with SAHA, induced less apoptosis in $p 53$ knockdown UM-PEL-1 cells as compared with that in mock cells (Figure 5G). In addition, the combination of nutlin-3 with Btz or SAHA induced more cell death as compared with that induced by either drug alone. Altogether, these results support a positive role for p53-induced apoptosis in mediating the antineoplastic effect of Btz in PEL.

Btz and SAHA synergize to induce KSHV lytic reactivation in $P E L$ xenografts, while Btz inhibits late lytic gene expression. Since the Btz/ SAHA combination induced reactivation of KSHV in UM-PEL-1c cells in culture (Figure 2A), we next examined this effect in vivo in UM-PEL-1 xenografts. While the transcription of latent genes was minimally affected by any treatment (Figure 6A), KSHV lytic reactivation was potently induced by SAHA and the Btz/SAHA combination in a gene-specific manner, beginning with the IE lytic switch RTA (Figure 6B). SAHA-induced IE transcripts $(K 8$, also known as $b$-Zip, and ORF45) were inhibited by concurrent Btz treatment (Figure 6B). Indeed, with the exception of LANA, RTA, and ORF21, the majority of SAHA-induced KSHV gene transcripts were almost uniformly inhibited in vivo by addition of Btz (Figure 6, B-D). Importantly, in contrast to the in vitro data showing disparate regulation of some late lytic genes, all late lytic genes examined in the in vivo UM-PEL-1 xenografts were consistently 
induced with SAHA but inhibited by addition of Btz (Figure 6D). Even though UM-PEL-1 cells are also coinfected with EBV, we did not observe significant EBV lytic gene reactivation by Btz or SAHA (Supplemental Figure 9). The consistent finding of the inhibition of all the tested late lytic viral transcripts suggested that Btz treatment, although effective in inducing lytic reactivation, was at the same time leading to a block in KSHV replicative cycle. To test this possibility, we examined the production of KSHV virus following SAHA and/or Btz treatment by measuring the amount of encapsidated extracellular viral DNA. In the presence of the lytic inducer SAHA, there was a 40-fold increase of encapsidated viral DNA as compared with that in untreated control (Figure 6E). This was in sharp contrast with Btz treatments that showed no increase in virion DNA in Btz-only-treated animals and totally abrogated SAHA-induced virion production in animals treated with Btz/SAHA combination.

Btz treatment blocks KSHV infectious virion production by UM-PEL-1c cells. Our in vivo results showed that the Btz/SAHA antitumor effect correlated with apoptosis and viral lytic induction occurring in the absence of late lytic transcription. The fact that lytic gene inhibition correlated with a lack of extracellular encapsidated viral DNA increase suggested that infectious viral production was blocked. This would be a desirable outcome in the clinical setting in treating PEL in the context of immunosuppression and AIDS. To assess the antiviral potential of these treatments, we examined the effect of Btz/SAHA on KSHV DNA replication and virus production in cultured UM-PEL-1c cells. UM-PEL1c cells were stimulated with Btz, SAHA, or Btz/SAHA, and the intracellular KSHV DNA load was measured at 72 hours by qPCR, and the infectivity of the supernatants was assessed by infection of 293 cells and LANA-based KSHV detection. UM-PEL-1c cells treated with either Btz alone or Btz/SAHA contained substantially higher intracellular viral DNA levels compared with the control or cells treated with SAHA only (Figure 7A). However, virus production decreased in both UM-PEL-1 cells treated with Btz alone and those treated with Btz/SAHA compared with that in cells treated with SAHA (Figure 7, B and C). Consistent with the results of Figure 6, we found that Btz abrogated the SAHAinduced production of infectious virions. Overall, these results demonstrate that, while both Btz and SAHA reactivate KSHV, Btz blocks mature virion production.

\section{Discussion}

An attractive option for treating PEL and other $\gamma$-herpesvirusinduced cancers is targeting endogenous latent viruses with drugs that reactivate their lytic replication, thereby eradicating virally infected reservoirs. In this study, using a direct xenograft PEL model, we demonstrated that the combination of the antineoplastic agents Btz and SAHA synergized to induce KSHV lytic replication, while leading to extensive apoptosis and a significant survival advantage in PEL-bearing mice. Importantly, this potent killing effect occurred in the absence of infectious KSHV production. Btz and SAHA are FDA-approved drugs that are clinically available and currently under investigation for the treatment of HIV and $\gamma$-herpesvirus-related lymphomas in National Cancer Institute-sponsored AIDS Malignancies Consortium clinical trials (AMC-053 and AMC-075).

Considering that all PEL tumors are infected with latent KSHV, the antineoplastic effect observed by the combined use of Btz and SAHA might be in part accomplished by their ability to target latency and induce KSHV lytic replication. While the mechanism by which Btz induces viral reactivation remains unclear, HDIs like SAHA are thought to induce $\gamma$-herpesvirus lytic reactivation through chromatin remodeling. HDACs regulate the transcriptional activity of $\operatorname{KSHV} \operatorname{RTA}(9,10)$. Viral lytic induction is known to cause $\mathrm{G}_{0} / \mathrm{G}_{1}$ cell cycle arrest, which can lead to cytotoxicity (37). Indeed, in UM-PEL-1 cells the percentage of apoptotic cells closely correlated with drug-induced lytic reactivation (vGPCR-positive cells), indicating that KSHV lytic replication could be causally associated with cytotoxicity. While single drugs alone tend not to induce robust KSHV lytic reactivation in PEL (38), the combination of Btz and SAHA synergized to induce KSHV lytic replication and enhanced apoptosis of PEL cells, an effect that translated into prolonged survival in vivo.

The powerful induction of lytic KSHV replication with concomitant inhibition of virus production was an unexpected yet clinically desirable outcome of the Btz/SAHA combination. To understand the nature of this inhibition, we analyzed viral DNA loads in vitro and found that Btz-treated UM-PEL-1c cells harbored nearly 4 fold the number of viral DNA copies compared with the control and SAHA-treated cells. We reasoned that since Btz was inhibiting late lytic gene expression, the accumulation of intracellular viral DNA could be a reflection of DNA replication, along with failure to complete the lytic replicative cycle. The results from viral infection assays support this hypothesis, as PEL cells stimulated with Btz or Btz/SAHA produced fewer infectious virions. This was confirmed in vivo: mice treated with Btz had markedly less encapsidated viral DNA in the ascites than those treated with SAHA alone.

The inhibition of infectious KSHV production by Btz is supported by a previous in vitro study describing Btz inhibition of virion production (39). This antiviral effect likely results from the dependency of KSHV on the proteasome throughout the viral replicative cycle, which has been described in the context of other herpesvirus $(40,41)$. Here, while Btz induced the expression of many KSHV lytic genes, the transcription of several key genes was affected negatively, indicating that the proteasome inhibition has gene-specific effects on viral lytic transcription. For instance, transcription of RTA and ORF45 was synergistically enhanced by combining Btz and SAHA, while SAHA-induced K8 expression was inhibited by Btz. This may be a significant event, as the K8 protein coordinately activates, along with RTA, the expression of some KSHV lytic genes (42). K8.1, a late lytic gene that encodes a key glycoprotein and is transcribed from the same locus as K8, was similarly found to be inhibited at the mRNA and protein levels. While we did not interrogate all ORFs encoded by KSHV, it is likely that there are other loci that are similarly inhibited by proteasome inhibition. As shown in Figures 2 and 6, we observed a distinct pattern of lytic activation between in vitro and in vivo experiments. This is most likely the consequence of differences between in vitro and host microenvironments, which are known to alter KSHV permissibility and viral gene expression in PEL (43). Finally, while lytic herpesviruses are canonically cytopathic, whether KSHV reactivation contributes to apoptosis in PEL when mature virion production is blocked remains to be elucidated. Although it has been previously reported that Btz can reactivate EBV expression (44), we did not observe reactivation of EBV, making it unlikely that EBV contributed to Btz/SAHA-induced cell death.

In addition to viral lytic induction, Btz and SAHA likely promote PEL cell apoptosis via other mechanisms. While the exact mechanisms of cell death by proteasome inhibitors remain con- 
troversial, studies have demonstrated that induction of cell death in B cell lymphomas by proteasome inhibitors is mediated by $\mathrm{p} 53$ (45-48). Similarly, a possible explanation for the Btz-induced apoptosis seen in our PEL model was posttranslational stabilization of phosphorylated and acetylated $\mathrm{p} 53$ protein along with the accumulation of its targets, p21 and Bax. The observed increase in phosphorylated $\mathrm{p} 53$ and $\gamma \mathrm{H} 2 \mathrm{AX}$ proteins by Btz is consistent with a DNA damage response (Figure 4A). The partial shRNAmediated abrogation of p53 expression in PEL xenografts, resulting in decreased cell death and blunted caspase activation, supports a role for p53 in mediating Btz-induced apoptosis in PEL. Additionally, the SAHA-induced p53 acetylation resulting in decreased p53-MDM2 interaction and augmented p21 transcription serves as evidence that p53 may also contribute to the antitumor effects of Btz/SAHA combination. While p53 acetylation is indispensable for its activation (35), the exact early events induced by SAHA and critical p53 acetylation sites in PEL remain to be determined in future studies. Last, histone hyperacetylation has been shown to have proapoptotic effects in other neoplastic models (30-32). The SAHA-induced acetylation and Btzmediated accumulation of acetylated histones that we observed likely contributed to chromatin remodeling and the activation of silenced viral and cellular genes.

In summary, the results from this study point to a novel treatment strategy for KSHV-infected PEL (Figure 7D). Using the Btz/ SAHA combination allows for robust viral induction while concurrently blocking infectious virus production, thus ensuring destruction of PEL cells. Given the observed anti-KSHV effect of Btz in stalling full lytic replication and virion production and the enhanced effect of Btz and SAHA on apoptotic pathways, this study provides a strong rationale for combining these drugs as a potent PEL therapy, especially in the setting of HIV and immunosuppression. Based on our findings, the clinical use of the combination of proteasome inhibitors and HDIs is clearly feasible for the treatment of PEL and potentially other $\gamma$-herpesvirus-related malignancies.

\section{Methods}

Reagents. Btz was obtained from Millennium Pharmaceuticals, Vorinostat (SAHA) was obtained from LC Laboratories, Nutlin-3 was obtained from Enzo Life Sciences, and cycloheximide was obtained from Sigma-Aldrich. The pan-caspase inhibitor (Z-VAD-FMK), caspase-9 inhibitor (Z-LEHDFMK), and caspase-8 inhibitor (Z-IETD-FMK) were purchased from MBL International. Primary antibodies to Bax, Bcl- $\mathrm{X}_{\mathrm{L}}, \mathrm{c}-\mathrm{MYC}, \mathrm{p} 21$, P-IкB- $\alpha$, caspase-8, acetyl-H3, total H3, acetyl p53 (Lys-382), phospho-p53 (Ser-15), GRP-78, GRP-94, P-eIF2 $\alpha$, total eIF2 $\alpha$, and CHOP were from Cell Signaling Technology; GAPDH, $\beta$-actin, IRF-4, c-FLIP, ubiquitin, and p53 were from Santa Cruz Biotechnology Inc.; total IKB- $\alpha, \mathrm{Bcl}-2$, CD30, and annexin VFITC were from BD Pharmingen; ATF- 6 was from Imgenex; and cIAP2 was from Abcam. YO-PRO-1 and PI were purchased from Invitrogen.

Cell lines. The UM-PEL-1 cell line was previously reported (14). Briefly, UM-PEL-1 cells were freshly isolated from malignant pleural effusion of a patient with PEL and transferred directly into the peritoneal cavities of NOD/SCID mice to avoid the changes in KSHV gene expression evident in cultured cells. For in vitro studies, UM-PEL-1c, a stable cell line established from UM-PEL-1, was cultured in RPMI 1640 (Mediatech) supplemented with 10\% FBS (Mediatech) and penicillin/streptomycin (Gibco BRL). The BC1 cell line $\left(\mathrm{KSHV}^{+} / \mathrm{EBV}^{+}\right)$, derived from an HIV-positive patient (49), and BC3 cell line $\left(\mathrm{KSHV}^{+} / \mathrm{EBV}^{-}\right)$, derived from an HIV-negative patient (50), were also cultured in RPMI 1640. HEK293 cells were cultured in DMEM (Mediatech) supplemented with 10\% FBS and penicillin/streptomycin.

PEL xenografts. All animal studies were conducted according to an approved IACUC protocol. The UM-PEL-1 model was established in NOD/SCID mice directly from a malignant pleural effusion of an elderly patient with PEL (14). UM-PEL-1 cells are positive for CD45, CD30, CD38, CD138, HLA-DR, HHV-8 (LANA1), and EBV-encoded RNA but negative for CD3, CD19, CD20, and CD79a (14). UM-PEL-1 cells display a complex karyotype and are monoclonal, based on IgG heavy chain gene rearrangement (14). UM-PEL-1 preserved its phenotype, IgG rearrangement, and mutation status in repeated animal experiments conducted over several years (data not shown). UM-PEL- 1 cells $\left(25 \times 10^{6}\right.$ cells) isolated from visible malignant ascites of UM-PEL-1 tumor-bearing mice were resuspended in $200 \mu \mathrm{l}$ ascites fluid and injected i.p. into NOD/SCID mice. On day 3 , mice were randomly assigned to DMSO $(50 \mu \mathrm{l}), \mathrm{Btz}(0.3 \mathrm{mg} / \mathrm{kg}$; twice a week), SAHA (60 mg/kg; daily), or Btz/SAHA treatment groups and treated i.p. for 3 weeks. Untreated mice exhibited visible ascites as early as day 5 . Mice were monitored daily and sacrificed when moribund or exhibiting signs of discomfort.

KSHV immunofluorescence and TUNEL assays. A total of $1 \times 10^{5}$ cells per treatment were cytospun at $66 \mathrm{~g}$ for 3 minutes and stained as previously described (51). The vGPCR and K8.1 antibodies (Advanced Biotechnologies) were used at 1:200 dilutions. The LANA antibody (Abcam) was used at 1:100 dilution. Alexa Fluor goat anti-rabbit (vGPCR), mouse (K8.1), and rat (LANA) were used as secondary antibodies (Invitrogen). Slides were fixed with ProLong Gold Antifade Reagent with DAPI (Invitrogen). MetaMorph 7.7 (marketed by Molecular Devices) was used to quantify K8.1/ $\mathrm{Cy}^{+}$cells, and data were normalized to $\mathrm{DAPI}^{+}$nuclei using a pixel-based approach (described in detail in the Supplemental Methods). All images were acquired using Zeiss AxioVision 4.8.2 with a Hamamatsu ORCA-R2 CCD camera and Zeiss Axiovert 200M inverted fluorescence microscope. TUNEL assay was performed as per manufacturer's instructions (In Situ Cell Death Detection Kit, Fluorescein, Roche).

Determination of $p 53$ half-life. UM-PEL- 1 cells $\left(25 \times 10^{6}\right.$ cells $)$ isolated from ascites of tumor-bearing mice were resuspended in $200 \mu$ lascites fluid and injected i.p. into NOD/SCID mice. At day 7 after injection, mice were treated i.p. with DMSO $(50 \mu \mathrm{l})$, Btz $(0.3 \mathrm{mg} / \mathrm{kg})$, SAHA $(60 \mathrm{mg} / \mathrm{kg})$, and Btz/SAHA $(0.3 \mathrm{mg} / \mathrm{kg} / 60 \mathrm{mg} / \mathrm{kg})$ and sacrificed after 24 hours. Cells harvested from the peritoneal effusions were treated with $50 \mu \mathrm{M}$ cycloheximide, and whole cell lysates extracted at $0,1,2,4$, and 8 hours after cycloheximide treatment were subjected to immunoblot analysis with an anti-p53 antibody. p53 protein levels were analyzed by densitometry and normalized to GAPDH.

p53 knockdown in UM-PEL-1 cells. The following plasmids were used for $p 53$ knockdown: p53 silencing lentiviral vector shp53pLKO.1 puro (Addgene plasmid no. 19119), control nonsilencing plasmid pLKO.1 TRC (Addgene plasmid no. 10879), and pseudovirus packaging plasmids psPAX2 and pMD2.G (Addgene Plasmid no. 12260 and no. 12259). Briefly, pseudovirus particles were generated by transfecting shp53pLKO.1 (3 $\mu \mathrm{g})$ or control plasmid $(3 \mu \mathrm{g})$ and each of the packaging plasmids $(6 \mu \mathrm{g})$ into $293 \mathrm{~T}$ cells in a T25 flask using $60 \mu \mathrm{l}$ of NovaFECTOR lipid transfection reagent (VennNova). Supernatant containing pseudovirus particles was harvested after 48 hours, filtered, and added to UM-PEL-1 cells in the presence of $10 \mu \mathrm{g} / \mathrm{ml}$ of polybrene for 8 hours. Pseudovirus supernatant was removed, and the transduced cells were cultured for 48 hours prior to the addition of $1.0 \mu \mathrm{g} / \mathrm{ml}$ puromycin for positive selection. To obtain stable expression, cells were maintained in $1.0 \mu \mathrm{g} / \mathrm{ml}$ puromycin for an additional 15 days prior to passage in NOD/SCID mice as described earlier.

Other experimental procedures. For details on immunoblotting, immunoprecipitation, real-time $\mathrm{qRT}$-PCR, proliferation, cell cycle, apopto- 
sis, virion production, viral DNA quantification, and EMSA see the Supplemental Methods.

Statistics. Statistical significance of the data was determined using the 2-tailed Student's $t$ test. $P$ values lower than 0.05 were considered significant. Statistical analysis was performed using QuickCals (Graph Pad Prism 5.0 software). Survival of animals was estimated using the Kaplan-Meier survival curve method, and differences in survival were calculated using the long-rank test (Graph Pad Prism).

\section{Acknowledgments}

This work was supported by the NIH Center for AIDS Research grant 5P30AI073961-05 (Core) (to J.C. Ramos and I.S. Lossos); NIH NCI2U01CA121947-04 (AIDS Malignancy Consortium), Leukemia \& Lymphoma Society (M0901391), and NIH NCI PO1CA-128115-01A2 grants (to J.C. Ramos); NIH CA109335 and NIH CA122105 grants and the Dwoskin Family and Recio Foundations (to I.S. Lossos); NIH NCI grant CA136387 (to E.A. Mesri); and by the University of Miami Sylvester Comprehensive Cancer Center. We would also like to acknowledge Krisztina Kovacs for assistance with optimizing the TUNEL assay, Xaibin Xi for spXBP1 primers, Lucas Cavallin for many of the viral primers, and
George McNamara, director of the analytical imaging core facility at the UMMSM Diabetes Research Institute, for expert assistance in analyzing immunofluorescence.

Received for publication April 27, 2012, and accepted in revised form February 28, 2013.

Address correspondence to: Juan Carlos Ramos, University of Miami, Sylvester Comprehensive Cancer Center, Division of Hematology and Oncology, 1475 NW 12th Ave (D8-4), Miami, Florida 33136, USA. Phone: 305.243.6611; Fax: 305.243.9161; E-mail: jramos2@med.miami.edu. Or to: Izidore S. Lossos, University of Miami, Sylvester Comprehensive Cancer Center, Division of Hematology and Oncology, 1475 NW 12th Ave (D8-4), Miami, Florida 33136, USA. Phone: 305.243.4785; Fax: 305.243.4787; E-mail: ilossos@med.miami.edu. Or to: Enrique A. Mesri, University of Miami, Sylvester Comprehensive Cancer Center, Department of Microbiology and Immunology, 1550 NW 10th Avenue, Papanicolaou Bldg., Room 109 (R138) Miami, Florida 33136, USA. Phone: 305.243.5659; Fax: 305.243.8309; E-mail: emesri@med.miami.edu.
1. Cesarman E, Chang Y, Moore PS, Said JW, Knowles DM. Kaposi's sarcoma-associated herpesvirus-like DNA sequences in AIDS-related body-cavity-based lymphomas. NEnglJ Med. 1995;332(18):1186-1191.

2. Chang $Y$, et al. Identification of herpesvirus-like DNA sequences in AIDS-associated Kaposi's sarcoma. Science. 1994;266(5192):1865-1869.

3. Cesarman E, Mesri EA. Pathogenesis of viral lymphomas. Cancer Treat Res. 2006;131:49-88.

4. Jones D, et al. Primary-effusion lymphoma and Kaposi's sarcoma in a cardiac-transplant recipient. NEngl J Med. 1998;339(7):444-449.

5. Carbone A, Cesarman E, Spina M, Gloghini A, Schulz TF. HIV-associated lymphomas and gamma-herpesviruses. Blood. 2009;113(6):1213-1224.

6. Boulanger E, et al. Prognostic factors and outcome of human herpesvirus 8-associated primary effusion lymphoma in patients with AIDS. J Clin Oncol. 2005;23(19):4372-4380.

7. Mesri EA, Cesarman E, Boshoff C. Kaposi's sarcoma and its associated herpesvirus. Nat Rev Cancer. 2010;10(10):707-719.

8. Grundhoff A, Ganem D. Inefficient establishment of KSHV latency suggests an additional role for continued lytic replication in Kaposi sarcoma pathogenesis. J Clin Invest. 2004;113(1):124-136.

9. Gwack Y, Byun H, Hwang S, Lim C, Choe J. CREBbinding protein and histone deacetylase regulate the transcriptional activity of Kaposi's sarcoma-associated herpesvirus open reading frame 50 . J Virol. 2001;75(4):1909-1917.

10. Sun R, Lin SF, Gradoville L, Yuan Y, Zhu F, Miller $G$. A viral gene that activates lytic cycle expression of Kaposi's sarcoma-associated herpesvirus. Proc Natl Acad Sci U S A. 1998;95(18):10866-10871.

11. Carraway HE, Gore SD. Addition of histone deacetylase inhibitors in combination therapy. JClin Oncol. 2007;25(15):1955-1956.

12. Xu WS, Parmigiani RB, Marks PA. Histone deacetylase inhibitors: molecular mechanisms of action. Oncogene. 2007;26(37):5541-5552.

13. Contreras X, et al. Suberoylanilide hydroxamic acid reactivates HIV from latently infected cells. J Biol Chem. 2009;284(11):6782-6789.

14. Sarosiek KA, et al. Efficacy of bortezomib in a direct xenograft model of primary effusion lymphoma. Proc Natl Acad Sci U S A. 2010;107(29):13069-13074.

15. Voorhees PM, Orlowski RZ. The proteasome and proteasome inhibitors in cancer therapy. Annu Rev Pharmacol Toxicol. 2006;46:189-213.
16. Siddiqi T, Joyce RM. A case of HIV-negative primary effusion lymphoma treated with bortezomib, pegylated liposomal doxorubicin, and rituximab. Clin Lymphoma Myeloma. 2008;8(5):300-304.

17. Obeng EA, Carlson LM, Gutman DM, Harrington WJ Jr, Lee KP, Boise LH. Proteasome inhibitors induce a terminal unfolded protein response in multiple myeloma cells. Blood. 2006;107(12):4907-4916.

18. Matta H, Chaudhary PM. The proteasome inhibitor bortezomib (PS-341) inhibits growth and induces apoptosis in primary effusion lymphoma cells. Cancer Biol Ther. 2005;4(1):77-82.

19. Keller SA, Schattner EJ, Cesarman E. Inhibition of NF-kappaB induces apoptosis of KSHV-infected primary effusion lymphoma cells. Blood. 2000;96(7):2537-2542.

20. Keller SA, et al. NF-kappaB is essential for the progression of KSHV-and EBV-infected lymphomas in vivo. Blood. 2006;107(8):3295-3302.

21. Bubman D, Guasparri I, Cesarman E. Deregulation of c-Myc in primary effusion lymphoma by Kaposi's sarcoma herpesvirus latency-associated nuclear antigen. Oncogene. 2007;26(34):4979-4986.

22. Liu J, Martin H, Shamay M, Woodard C, Tang QQ, Hayward SD. Kaposi's sarcoma-associated herpesvirus LANA protein downregulates nuclear glycogen synthase kinase 3 activity and consequently blocks differentiation. J Virol. 2007;81(9):4722-4731.

23. Friborg J Jr, Kong W, Hottiger MO, Nabel GJ. p53 inhibition by the LANA protein of KSHV protects against cell death. Nature. 1999;402(6764):889-894.

24. Lambert PF, Kashanchi F, Radonovich MF, Shiekhattar R, Brady JN. Phosphorylation of p53 serine 15 increases interaction with CBP. J Biol Chem. 1998;273(49):33048-33053.

25. Chehab NH, Malikzay A, Stavridi ES, Halazonetis TD. Phosphorylation of Ser-20 mediates stabilization of human p53 in response to DNA damage. Proc Natl Acad Sci U S A. 1999;96(24):13777-13782.

26. Vogelstein B, Lane D, Levine AJ. Surfing the p53 network. Nature. 2000;408(6810):307-310.

27. el-Deiry WS, et al. WAF1, a potential mediator of p53 tumor suppression. Cell. 1993;75(4):817-825.

28. Giaccia AJ, Kastan MB. The complexity of p53 modulation: emerging patterns from divergent signals. Genes Dev. 1998;12(19):2973-2983.

29. Richon VM, et al. A class of hybrid polar inducers of transformed cell differentiation inhibits histone deacetylases. Proc Natl Acad Sci U S A. 1998; 95(6):3003-3007.
30. Miller CP, Rudra S, Keating MJ, Wierda WG, Palladino M, Chandra J. Caspase-8 dependent histone acetylation by a novel proteasome inhibitor, NPI0052: a mechanism for synergy in leukemia cells. Blood. 2009;113(18):4289-4299.

31. Sato A, Asano T, Ito K, Sumitomo M, Asano T. Suberoylanilide hydroxamic acid (SAHA) combined with bortezomib inhibits renal cancer growth by enhancing histone acetylation and protein ubiquitination synergistically. BJU Int. 2012;109(8):1258-1268.

32. Kikuchi J, et al. Histone deacetylases are critical targets of bortezomib-induced cytotoxicity in multiple myeloma. Blood. 2010;116(3):406-417.

33. Ito A, et al. MDM2-HDAC1-mediated deacetylation of $\mathrm{p} 53$ is required for its degradation. $Е M B O J$. 2002;21(22):6236-6245

34. Honda R, Tanaka H, Yasuda H. Oncoprotein MDM2 is a ubiquitin ligase E3 for tumor suppressor p53. FEBS Lett. 1997;420(1):25-27.

35. Tang Y, Zhao W, Chen Y, Zhao Y, Gu W. Acetylation is indispensable for p53 activation. Cell. 2008; 133(4):612-626.

36. Sarek G, et al. Reactivation of the $\mathrm{p} 53$ pathway as a treatment modality for KSHV-induced lymphomas. J Clin Invest. 2007;117(4):1019-1028.

37. Wu FY, et al. Lytic replication-associated protein (RAP) encoded by Kaposi sarcoma-associated herpesvirus causes p21CIP-1-mediated G1 cell cycle arrest through CCAAT/enhancer-binding protein-alpha. Proc Natl Acad Sci U S A. 2002;99(16):10683-10688.

38. Yu Y, Black JB, Goldsmith CS, Browning PJ, Bhalla K, Offermann MK. Induction of human herpesvirus-8 DNA replication and transcription by butyrate and TPA in BCBL-1 cells. J Gen Virol. 1999;80(pt 1):83-90.

39. Brown HJ, McBride WH, Zack JA, Sun R. Prostratin and bortezomib are novel inducers of latent Kaposi's sarcoma-associated herpesvirus. Antivir Ther. 2005;10(6):745-751.

40. Randow F, Lehner PJ. Viral avoidance and exploitation of the ubiquitin system. Nat Cell Biol. 2009;11(5):527-534.

41. Klass CM, Krug LT, Pozharskaya VP, Offermann MK. The targeting of primary effusion lymphoma cells for apoptosis by inducing lytic replication of human herpesvirus 8 while blocking virus production. Blood. 2005;105(10):4028-4034.

42. Lefort S, Flamand L. Kaposi's sarcoma-associated herpesvirus K-bZIP protein is necessary for lytic viral gene expression, DNA replication, and virion production in primary effusion lymphoma cell 
lines. J Virol. 2009;83(11):5869-5880.

43. Staudt MR, Kanan Y, Jeong JH, Papin JF, HinesBoykin R, Dittmer DP. The tumor microenvironment controls primary effusion lymphoma growth in vivo. Cancer Res. 2004;64(14):4790-4799.

44. Fu DX, et al. Virus-associated tumor imaging by induction of viral gene expression. Clin Cancer Res. 2007;13(5):1453-1458.

45. Yu D, Carroll M, Thomas-Tikhonenko A. p53 status dictates responses of $\mathrm{B}$ lymphomas to monotherapy with proteasome inhibitors. Blood. 2007; 109(11):4936-4943.

46. Concannon CG, et al. Apoptosis induced by proteasome inhibition in cancer cells: predominant role of the p53/PUMA pathway. Oncogene. 2007; 26(12):1681-1692.

47. Pandit B, Gartel AL. Proteasome inhibitors induce p53-independent apoptosis in human cancer cells. Am J Pathol. 2011;178(1):355-360.

48. Perez-Galan P, Roue G, Villamor N, Montserrat E, Campo E, Colomer D. The proteasome inhibitor bortezomib induces apoptosis in mantle-cell lymphoma through generation of ROS and Noxa activation independent of p53 status. Blood. 2006; 107(1):257-264.

49. Cesarman E, Moore PS, Rao PH, Inghirami G, Knowles DM, Chang Y. In vitro establishment and characterization of two acquired immunodefi- ciency syndrome-related lymphoma cell lines (BC-1 and BC-2) containing Kaposi's sarcoma-associated herpesvirus-like (KSHV) DNA sequences. Blood. 1995;86(7):2708-2714.

50. Arvanitakis L, et al. Establishment and characterization of a primary effusion (body cavity-based) lymphoma cell line (BC-3) harboring kaposi's sarcoma-associated herpesvirus (KSHV/HHV-8) in the absence of Epstein-Barr virus. Blood. 1996; 88(7):2648-2654.

51. Mutlu AD, et al. In vivo-restricted and reversible malignancy induced by human herpesvirus- 8 KSHV: a cell and animal model of virally induced Kaposi's sarcoma. Cancer Cell. 2007;11(3):245-258. 